International Journal of Medical Sciences

ISSN 1449-1907 www.medsci.org 2008 5(1):9-17

Research Paper

(C) Ivyspring International Publisher. All rights reserved

\title{
Does eGFR improve the diagnostic capability of S-Creatinine concentration results? A retrospective population based study
}

\author{
Anders Kallner ${ }^{1}$, Peter A Ayling 2, Zahra Khatami ${ }^{2}$ \\ 1. Department of Clinical Chemistry, Karolinska University Hospital, SE 17176, Stockholm, Sweden \\ 2. Department of Biochemistry, Queen's Hospital, Romford, Essex, RM70AG UK
}

Correspondence to: Anders Kallner, Associate Professor, MD, PhD, Phone +46 85177 4943; Fax +46 85177 2899; e-mail: anders.kallner@ki.se

Received: 2007.11.14; Accepted: 2008.01.03; Published: 2008.01.05

The use of MDRD-eGFR to diagnose Chronic Kidney Disease (CKD) is based on the assumption that the algorithm will minimize the influence of age, gender and ethnicity that is observed in S-Creatinine concentration and thus allow a single cut-off at which further diagnostic and therapeutic actions should be considered. This hypothesis is tested in a retrospective analysis of outpatients $(\mathrm{N}=93,404)$ and hospitalised $(\mathrm{N}=35,572)$ patients in UK and Sweden, respectively. An algorithm based on the same model as the MDRD-eGFR algorithm was derived from simultaneously measured S-Creatinine concentrations and Iohexol GFR in a subset of 565 patients. The combined uncertainty of using this algorithm was estimated to about $15 \%$ which is about three times that of the S-Creatinine concentration results. The diagnostic performance of S-Creatinine concentration was evaluated using the Iohexol clearance as the reference procedure. It was shown that the diagnostic capacity of MDRD-eGFR, as it stands, has no added value compared to S-Creatinine. The gender and age differences of the S-Creatinine concentrations in the dataset persist after applying the MDRD-eGFR algorithm. Thus, a general use of the MDRD-eGFR does not seem justified. Furthermore the claim that the eGFR is adjusted for body area is misleading; the algorithm does not include any body size marker. It is thus a dangerous marker for guiding drug administration.

Key words: CKD, Diagnosis, algorithm, outpatients, inpatients

\section{Introduction}

Measurement of the S-Creatinine concentration ${ }^{1}$ is one of the most frequently requested tests in the biochemistry laboratory. Most of the requests may not necessarily be related to chronic kidney disease or a specific investigation of renal function [1,2]. Never the less, health authorities in several countries have ruled that each S-Creatinine result shall be accompanied by a quantity that is calculated from the S-Creatinine, the age and, if applicable, modified for gender (female) and ethnicity (Afro-American). This quantity is called eGFR 2 ; (estimated glomerular filtration rate). The most frequently used algorithm is the 4-parameter MDRD algorithm $[3,4,5]$. It has been reported that this data

\footnotetext{
${ }^{1}$ A note on terminology: The quantity measured is S-Creatinine; amount of substance concentration. In the text this is abbreviated to S-Creatinine.

${ }^{2}$ Abbreviations: S-Creatinine: S-Creatinine; amount of substance concentration $(\mu \mathrm{mol} / \mathrm{L})$. Pt-Iohexol: Patient-Iohexol elimination; rate $\mathrm{mL} /\left(\min \times 1.73 \mathrm{~m}^{2}\right)$. MDRD-eGFR estimated Glomerular Filtration Rate using the 4-parameter MDRD equation. eGFR II estimated Glomerular Filtration Rate using the presently derived equation. LIS: Laboratory Information System.
}

transformation enhances the diagnosis of chronic kidney disease (CKD) as a surrogate marker for glomerular filtration rate and is superior to S-Creatinine. It is further suggested that the algorithm allows a single cut-off value for the diagnosis of CKD, particularly stage III [4]. Considering the physiological age and gender changes of S-Creatinine the algorithm therefore needs to neutralize these effects.

To validate this hypothesis we present a retrospective study in which we apply the MDRD-eGFR algorithm to results from primary health care in the United Kingdom (UK) and hospitalized patients in Sweden (SE). We also derived a 4-term algorithm based on the same model as the MDRD algorithm using simultaneously measured S-Creatinine and Iohexol GFR. The present study thus focuses on a comparison of the diagnostic performance of eGFR and S-Creatinine, estimating the uncertainty of the eGFR and testing the transferability of the eGFR between sites. 


\section{Methods and materials}

\section{Database}

Data from UK included all S-Creatinine results from the primary care of the BHR NHS trust (Essex) during 2005 (women 49,169, men 44,235). Data from SE included all S-Creatinine results from inpatients of the Karolinska University Hospital (KS) in Stockholm (women 14,124, men 21,648) during a one year period.

Results from patients above 19 years with S-Creatinine between $70 \mu \mathrm{mol} / \mathrm{L}$ and $200 \mu \mathrm{mol} / \mathrm{L}$ were partitioned according to gender and age (Table 1 and Figure 1). The age was calculated from the year of birth to the year of sampling, irrespective of dates of birth or sampling. The population in neither of the catchment areas allowed singling out a group of African origin and no record of ethnicity, was registered in the databases.

Results from all patients in whom S-Creatinine and Pt-Glomerular filtration rate (Pt-Iohexol) were measured on the same day were obtained from the LIS of KS and those fulfilling the inclusion criteria chosen $(\mathrm{N}=565)$. Results were partitioned according to age and gender (Table 1).

Since all tags that could uniquely identify a patient were removed - only age and gender were retained - the study did not require permission from the Ethics committees.
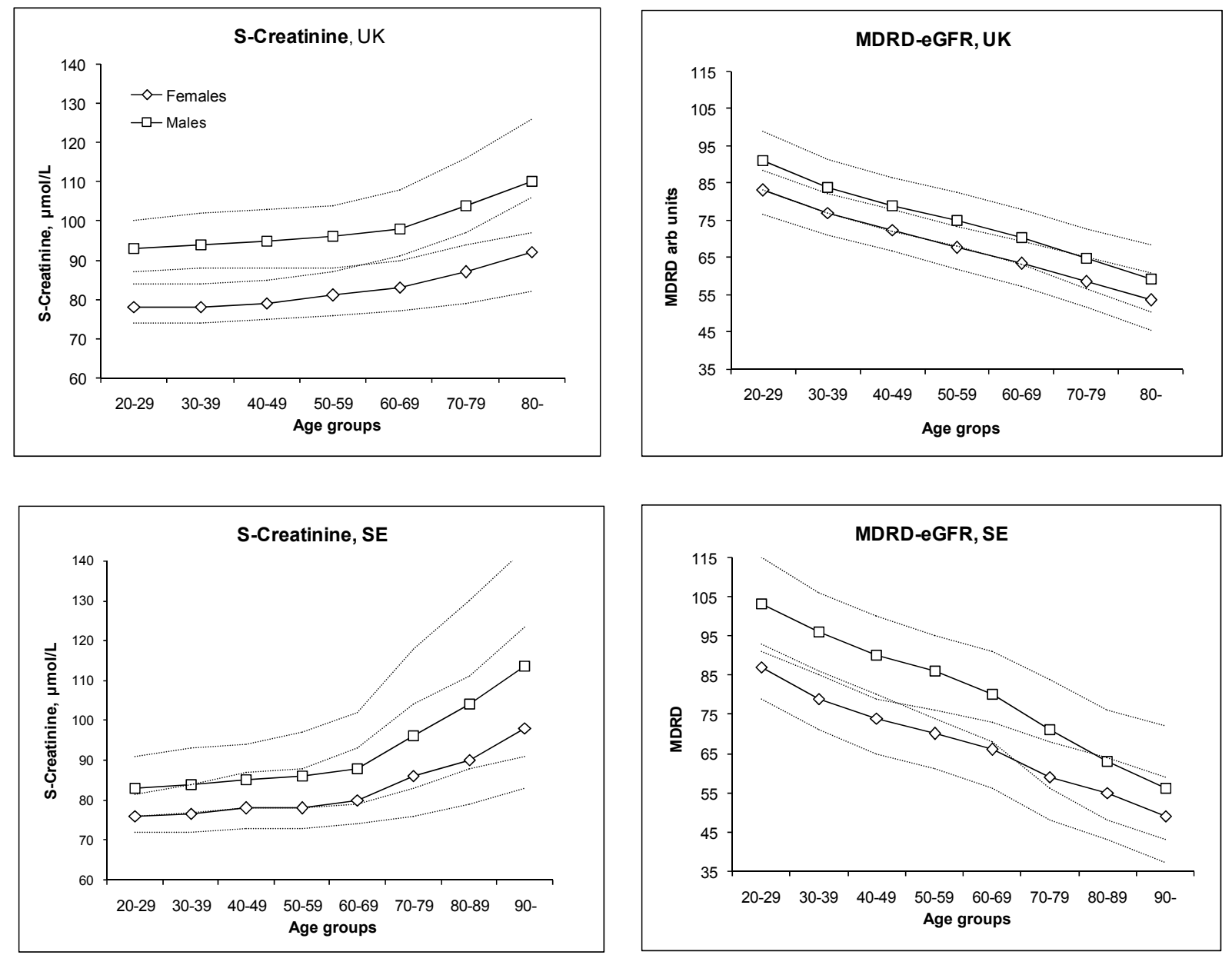

Figure 1. The age-dependent changes of S-Creatinine and MDRD-eGFR for females and males in the Swedish and United Kingdom cohorts. Triangles represent females, diamonds males. 
Table 1 Partitioning of data. Group concentrations and e-GFR values are given as medians and the 25 - 75 percentile interval.

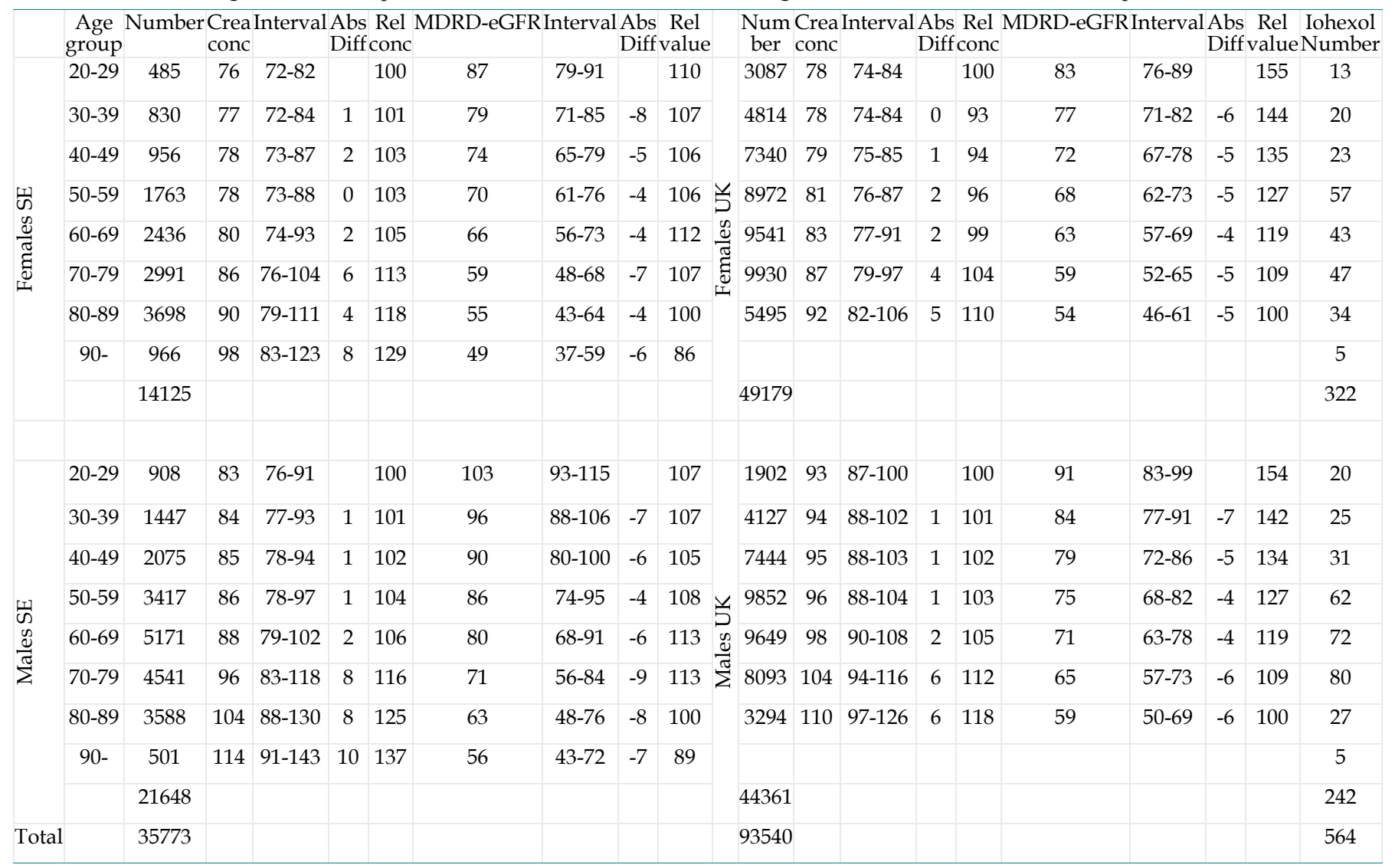

\section{Analytical}

\section{Creatinine}

SE. S-Creatinine were measured using Beckman LX20 instruments, calibrators and reagents with a modified kinetic Jaffe method. The system was monitored by routine IQC procedures and participation in Equalis EQA system. The laboratory reports a measurement uncertainty of $5 \%(k=1$, i.e. 1 $\mathrm{SD})$ over the entire reporting interval. The laboratory was accredited according to the EN/ISO 15189.

UK. S-Creatinine were measured using Olympus 640 analysers. Reagents and calibrators for a modified kinetic Jaffe method were obtained from Olympus Diagnostics Ltd UK. The quality of results was monitored through-out the period by IQC (Randox Laboratories Ltd, Ireland) procedures and participation in UK NEQAS. The laboratory reports a measurement uncertainty of $5 \%$. The laboratory was accredited according to CPA (UK).

Since there is no prerequisite in the guidelines that laboratories shall have harmonized their results beyond using a traceable calibrator to abide by the recommended cut-off the acceptance by the EQAS was regarded as sufficient to disregard any bias.

\section{lohexol clearance (Pt-lohexol)}

Omnipaque ${ }^{\circledR}, 5 \mathrm{~mL}$, was injected intravenously to fasting, well hydrated patients. Samples were drawn before and at 230-240 minutes after the injection. The Iohexol concentration was measured by HPLC on a $\mathrm{C}_{18}$ column (Zorbax SB-18, Chromtech, USA) eluted with Methanol/phosphoric acid and assayed using Waters 2487 absorbance detector and 2795 Separation Module. The system was calibrated with Iohexol dissolved in control serum (Autonorm, Sero AS, Oslo, Norway). Iopamiro (Astra Zeneca, Södertälje, Sweden) was used as internal standard (IS). Typically, the IS was eluted twice as fast as Iohexol and baseline separation was achieved. Measurement uncertainty was $3.2 \%$. The chromatograms were digitized and the Iohexol clearance was estimated using a one-point method [5,6].

\section{Calculation of MDRD-eGFR and nonlinear fitting}

The eGFR of the UK and SE results were calculated using the 4 variable version [4] of the MDRD equation. Since UK and SE express S-Creatinine in $\mu \mathrm{mol} / \mathrm{L}$ the conversion factor $1 / 88.4$ was incorporated in the original MDRD formula to allow direct use of S-Creatinine expressed in $\mu \mathrm{mol} / \mathrm{L}$.

The S-Creatinine, age and gender were fitted to 
the measured Pt-Iohexol obtained from the SE results; the data set is specified in table 2. The Marquardt-Levenberg iterative algorithm that is available in SigmaStat was used to model the algorithm similar to the MDRD-eGFR algorithm The 'constants' derived for the males were fitted, together with a variable factor, to the female Pt-Iohexol values. Thus, an algorithm was derived with an 'if female' factor of 0.82 different from 0.74 stated in the MDRD-eGFR algorithm.

It is reasonable to assume that the fitting is less accurate at the extremes of the measuring interval.

Table 2. Specifications of the cohorts used to derive the eGFR II algorithm. Pt-Iohexol in $\mathrm{mL} /\left(\min \times 1.73 \mathrm{~m}^{2}\right)$

\begin{tabular}{|c|c|c|}
\hline & Men & Women \\
\hline Number & 323 & 242 \\
\hline Age median & 63 & 62 \\
\hline $25 \%$ percentile & 50,8 & 52 \\
\hline $75 \%$ percentile & 73 & 75 \\
\hline Pt Iohexol median & 63 & 52 \\
\hline 25\% percentile & 48 & 36 \\
\hline 75\% percentile & 78 & 71 \\
\hline Pt Iohexol Max & 136 & 120 \\
\hline Pt-Iohexol Min & 16 & 15 \\
\hline
\end{tabular}

ROC analysis

Using the SE database of Pt-Iohexol as reference, the clinical sensitivity, specificity and likelihood ratios were calculated for a threshold cut-off of $60 \mathrm{~mL} /(\mathrm{min} \mathrm{x}$ $\left.1.73 \mathrm{~m}^{2}\right)$ for the MDRD-eGFR and 95 and $115 \mu \mathrm{mol} / \mathrm{L}$ for S-Creatinine in women and men, respectively (equating to the upper limits of the reference intervals recommended by the laboratory at the time of the study. It should be pointed out, however, that the reference values of the laboratory are just reference values estimated as the mean $+2 \mathrm{SD}$ of the reference population and not meant as action limits).

Statistics

The databases and graphs were created with Microsoft EXCEL. As appropriate, JMP v 5.1 (SAS Institute, Cary NC, USA) and SigmaPlot/Sigmastat v
10 and 3, respectively (Systat Software, GmbH, Erkrath, Germany) were used. Normality was tested by the Kolmogorov-Smirnov test. Comparisons between results were evaluated using the Mann-Whitney rank sum test.

\section{Results}

Differences between age groups for S-Creatinine and MDRD-eGFR results in men and women were evaluated by the Kruskal-Wallis "one-way ANOVA by rank" followed by the Dunn's multiple comparison procedure. All comparisons of the MDRD results showed a significant difference between age groups whereas the S-Creatinine in the three lowest age groups was not significantly different in the SE cohort nor the two lowest in the UK cohort (Figure 1).

The difference between the creatinine results obtained in the UK and SE is small for women but about $10 \mu \mathrm{mol} / \mathrm{L}$ for men (Table 1 ).

The medians of S-Creatinine were significantly different between the genders and this difference was retained in the MDRD-eGFR values $(p<0.001)$. The ratio of the medians of S-Creatinine and MDRD-eGFR between women and men decreased from 0.91 for S-Creatinine to 0.79 for MDRD-eGFR in the SE cohort and from 0,84 for S-Creatinine to 0.72 for MDRD-eGFR in the UK cohort. This indicates that in both cohorts the difference between genders is increased by the algorithm. It may be pointed out that the ratio between the reference values of S-Creatinine for women and men used in the laboratory (SE) was 0.82 .

The derived algorithm (table 3 row 4) was applied to the creatinine and age data from SE and UK to calculate the "eGFR II". The difference between the eGFR II and the MDRD-eGFR was statistically significant in all age groups and both cohorts except in the highest age groups in both the SE and UK cohorts (Table 4). The largest difference between medians of the groups were $11 \mathrm{~mL} /\left(\min \times 1.73 \mathrm{~m}^{2}\right)$ and 9 $\mathrm{mL} /\left(\min \times 1.73 \mathrm{~m}^{2}\right)$, recorded in the youngest age-groups of the UK and SE cohorts of females, respectively. This indicates that results of the generally recommended algorithm and a locally derived algorithm will give different results.

Table 3. Constants and exponents obtained by non-linear fitting of S-Creatinine results to Pt-Iohexol as the dependent variable. Row 1 summarizes the original MDRD algorithm, rows 2 and 3 those obtained in the SE study, row 4 when the expression in row 2 is adjusted to that in row 3 by introducing an 'if female' factor and row 5 the algorithm obtained considering both men and women.

\begin{tabular}{|c|c|c|c|c|c|c|c|c|c|c|c|}
\hline & & $\mathrm{N}$ & $\begin{array}{c}\text { Constant } \\
\text { (Mass } \\
\text { units) }\end{array}$ & SEM & $\begin{array}{l}\text { Exp } \\
\text { crea }\end{array}$ & SEM & Exp age & SEM & 'If female' & SEM & $\mathrm{R}^{2} \mathrm{Adj}$ \\
\hline 1 & MDRD-eGFR & 1628 & 186 & --- & $-1,154$ & --- & $-0,203$ & --- & 0,742 & --- & --- \\
\hline 2 & Women & 242 & 297 & 53 & -0.918 & 0.068 & -0.382 & 0.045 & & & 0.617 \\
\hline 3 & Men & 323 & 311 & 31 & -0.852 & 0.043 & -0.344 & 0.026 & & & 0.707 \\
\hline 4 & Women & 242 & 311 & & -0.852 & & -0.344 & & 0.821 & 0.012 & 0.617 \\
\hline 5 & All & 565 & 321 & 33 & -0.813 & 0.040 & -0.375 & 0.026 & & & 0.608 \\
\hline
\end{tabular}


The medians in the age groups of Pt-Iohexol, S-Creatinine and eGFR II are shown in figure 2. This material comprised 242 females and 323 males (Table 1). For clarity the creatinine concentration is expressed as 10,000/S-Creatinine. This figure illustrates the parallelism between the markers.

An uncertainty budget [8] was established to estimate the combined uncertainty of the eGFR II calculations. S-Creatinine of $100 \mu \mathrm{mol} / \mathrm{L}$ equates to an MDRD-eGFR of about $60 \mathrm{~mL} /\left(\min \times 1.73 \mathrm{~m}^{2}\right)$. The standard uncertainties of the factors and exponents obtained in the fitting of the S-Creatinine to the Pt-Iohexol (Table 3) were used. The uncertainty of the creatinine results was assumed to be $5 \%$. The major sources of the combined uncertainty were S-Creatinine $(7 \%)$, the factor $(321 ; 42 \%)$, the derived exponent for creatinine $(-0.813 ; 1 \%)$, the exponent for the age $(-0,375 ; 49 \%)$ and the 'if female' factor $(1 \%)$. The combined uncertainty was about $15 \%$ resulting in an interval of the expanded uncertainty $(k=2)$ from 42 $\mathrm{mL} /\left(\min \times 1.73 \mathrm{~m}^{2}\right)$ to $78 \mathrm{~mL} /\left(\min \times 1.73 \mathrm{~m}^{2}\right)$. The statistically significant minimal difference between observations (reference difference) at a calculated

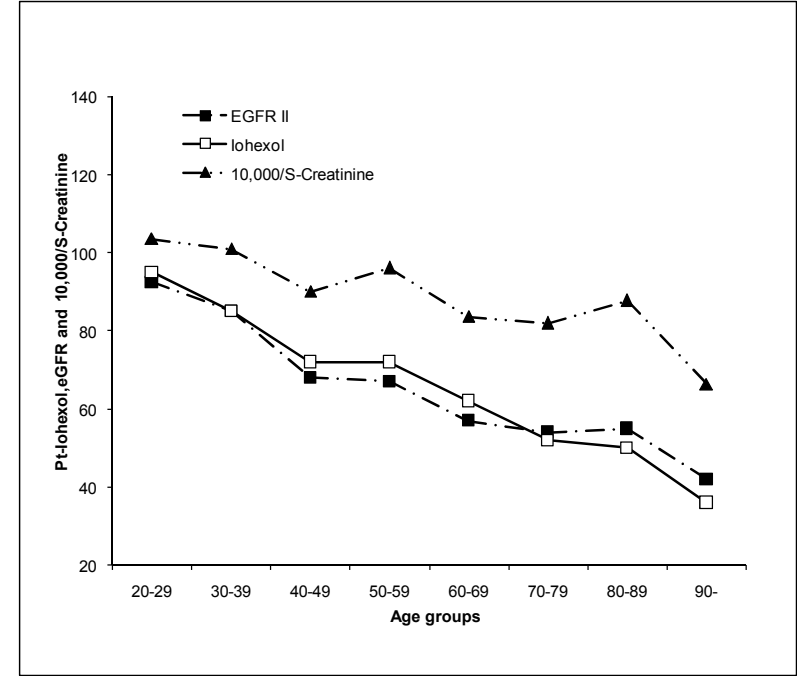

Figure 2. From top to bottom the inverse S-Creatinine (10000/S-Creatinine, filled triangles), Pt-Iohexol (filled diamonds, solid line) and eGFR II (filled squares) of the SE Iohexol data set (women in the right panel).
MDRD-eGFR of $60 \mathrm{~mL} /\left(\min \times 1.73 \mathrm{~m}^{2}\right)$ is thus $\Delta_{\text {sign }}=z \times(60 \times 0.15) \times \sqrt{2} \approx z \times 13 \mathrm{~mL} /(\min \times 1.73$ $\left.\mathrm{m}^{2}\right) \cdot[9]$ minimal significant difference between two observations is thus about $26 \mathrm{~mL} /\left(\min \times 1.73 \mathrm{~m}^{2}\right)$ or 43 $\%$ of the decision value. The corresponding minimal difference between S-Creatinine observations is about $14 \quad \mu \mathrm{mol} / \mathrm{L} \quad(14$ $\Delta_{\text {sign }}=2 \times(100 \times 0.05) \times \sqrt{2} \approx 2 \times 7 \mu \mathrm{mol} / \mathrm{L}$

ROC curves were calculated (Figure 3 ) for the S-Creatinine and eGFR. At the suggested cut-offs, Pt-Iohexol of $60 \mathrm{~mL} /\left(\min \times 1.73 \mathrm{~m}^{2}\right)$ and S-Creatinine 95 and $115 \mu \mathrm{mol} / \mathrm{L}$ (the upper reference limits of the laboratory), respectively, the likelihood ratio (LR) was 4.4, 3.6, 1.7 and 3.1 for S-Creatinine in men and women and MDRD-eGFR, respectively. The z-scores adjusted with Yates correction indicate a difference in favor of S-Creatinine between the LR of S-Creatinine and eGFR. This difference is statistically significant for men but not for women ( $\mathrm{z}=2.2$ and 1.4, respectively).

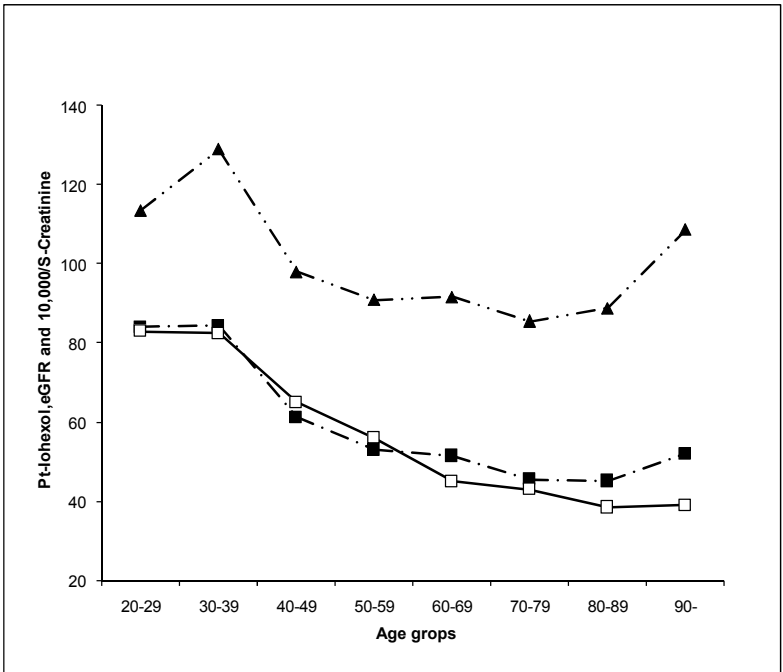

At a level of confidence of $95 \%, z=2$ and the 

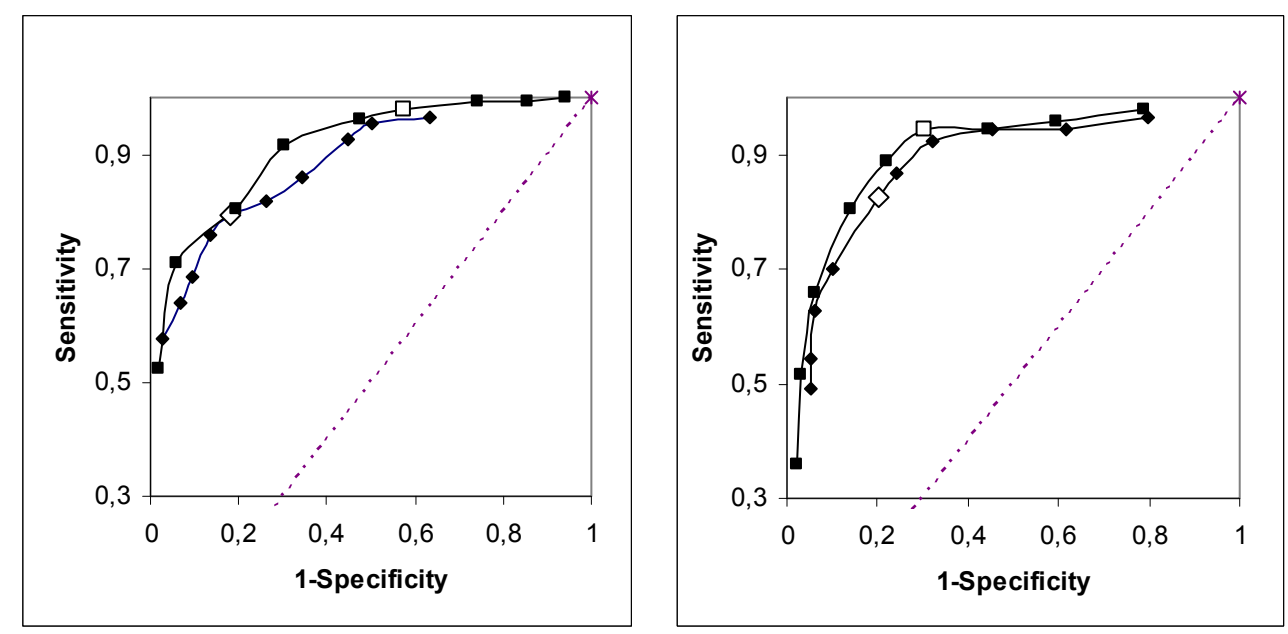

Figure 3. Partial ROC curves. The left panel is based on 342 men, the right on 242 women. Squares refer to S-Creatinine and diamonds to eGFR II. Open symbols refer to the predetermined reference limits and cut-offs.

\section{Discussion}

A recent study reported the performance of MDRD-eGFR in relation to measured GFR in a large diverse population [10]. The present study focuses on how the MDRD-eGFR performs in similar cohorts in relation to S-Creatinine which is the primary, measured quantity. This relation has been poorly studied but two independent studies were recently published [11,12].

Traditionally the kidney function has been estimated by the glomerular function through "creatinine clearance" although this procedure has long been questioned [13]. Major reasons for the concern are that creatinine is continuously generated; it is secreted and reabsorbed from the tubules and excreted by the intestine. In particular the practical problems with urine collection are difficult to avoid. S-Creatinine varies with age, muscle mass, diet and exercise and differs between genders. Other estimates of GFR have been based on exogenous substances e.g. Inulin and Iohexol. Analytically, the much used Jaffe method is liable to interferences by both endogenous, e.g. ketone bodies, and exogenous substances e.g. certain drugs. The use of a kinetic modification of the Jaffe assay has diminished these problems. Enzymatic methods, HPLC methods and ID-MS methods are available but may be too costly for routine application in most laboratories.

The calibration of S-Creatinine measurements has been a major concern [14] and a special factor in the MDRD-eGFR algorithm has been derived for calibrators that have been assayed by ID-MS [15]. Different MDRD-eGFR algorithms are thus in use. This will cause an indirect additional increase of the interlaboratory uncertainty of the eGFR [16]. The trueness of measurements is an often neglected problem in formulating common cutoff values, set-point values or recommendations. Myers et al. [14] concluded that "even if the imprecision is low and the assay is standardized to an ID-MS reference measurement procedure, if analytical non-specificity bias remains, then errors in estimated GFR for individual patients will occur".

Although the uncertainty contribution by S-Creatinine is small this does not mean that changes in the calibration of S-Creatinine can be disregarded.

Accredited laboratories participate in External Quality Assessment Schemes (EQAS) or Proficiency Testing (PT) that are designed to assess the trueness of measurements. The only measurement in eGFR is creatinine; therefore EQAS will only evaluate the measurement of creatinine, not the calculated quantity.

The analytical "sensitivity" of S-Creatinine is slightly larger than that for MDRD-eGFR, thus if S-Creatinine changes from 90 to $115 \mu \mathrm{mol} / \mathrm{L}$, i.e. 25 units, then the MDRD-eGFR will decrease from 82 to $61 \mathrm{~mL} /\left(\min \times 1.73 \mathrm{~m}^{2}\right)$, i.e. 21 units.

Estimated glomerular filtration rate, eGFR, is claimed to eliminate some of the disadvantages of S-Creatinine and 'creatinine clearance'. Many algorithms for eGFR include e.g. S-Albumin, S-Urea, and patient weight. Thorough evaluations and comparisons have been published with extensive accounts of kidney function $[3,4,5]$ including a healthy cohort $[17,18]$. The professions have favored the 4-parameter MDRD [4] algorithm that is based on only S-Creatinine, the patient's age, gender and ethnicity. It can be described as the reciprocal of S-Creatinine enhanced by multiplying with the reciprocal of the age and, if appropriate, adjusted by a factor for the gender and ethnicity. An additional factor adjusts the result numerically to the order of magnitude of Pt-Iohexol. The unit embedded in this factor $\left(\mathrm{mL} /\left(\min \times 1.73 \mathrm{~m}^{2}\right)\right.$ 
formally adjusts the dimension of the calculated number to that of clearance. The algorithm does not include any reference to the size (body area) of the actual patient. As a result a $2 \mathrm{~m}$ and $100 \mathrm{~kg}$ and a $165 \mathrm{~m}$ $50 \mathrm{~kg}$ individual of the same age and S-Creatinine would have the same MDRD-eGFR expressed in $\mathrm{mL} /\left(\min \times 1.73 \mathrm{~m}^{2}\right)$. It is important to understand that the regression function may hold true on a population basis but not in an individual. The use of eGFR in the individual case, after due adjustment for the body size, may therefore still be misleading in adjusting the dosage of drugs. An unexplored factor may be the know anthropometrical differences between Americans and others. This is an additional source of uncertainty in the use of MDRD-eGFR.

Only one cut-off value for MDRD-eGFR of 60 $\mathrm{mL} /\left(\min \times 1.73 \mathrm{~m}^{2}\right)$ (CKD stage III) is recommended by the NKDEP [4], for all ages and both sexes, below which additional investigations of the kidney function should be initiated. Thus the NKDEP assumes that the physiological changes of S-Creatinine by age and gender will be neutralized by the algorithm. Our results unequivocally show that this is not the case (Figure 1). The age dependency of MDRD-eGFR was at least of the same order of magnitude as that of S-Creatinine. Similar results, a decrease of about $7 \%$ per decade were recently reported [19]. The K/DOQI report [3] suggests a decrease in the GFR with about 1 $\mathrm{mL} / \mathrm{min}$ per year of age above 20 years. Our data shows that this is not eliminated by the MDRD-eGFR. Thus a common cut-off is not applicable to all ages.

The K/DOQI report further suggests $8 \%$ lower
GFR [3] values in women than in men but the original MDRD-eGFR algorithm suggests a factor of 0.741 . Our Iohexol study gives a factor of $0.82 \pm 0.01$ (SEM) which is also lower than that expected from the DOQI report $(0,92)$. The difference in the ratio between females and males of S-Creatinine $(0,91)$ and MDRD $(0,79)$ shows that the gender dependence of the markers increases in the MDRD-eGFR rather than being reduced or eliminated.

The difference in S-Creatinine between the SE and UK cohorts can in part be due to the difference in the patient types. Considering the equality between the results obtained in SE and UK women it is less likely that the difference is due to measurement bias. The problem of trueness will necessarily be aggravated by introduction of an algorithm in which constants and exponents have been derived at a location other than that in which it is used. The algorithms estimated in the present study were established by fitting data to the same model as the original MDRD-eGFR and the resulting coefficients and exponents are different (Table 3) - but the difference in calculated results are not clinically important (Table 4 ) between adjacent age groups in view of the uncertainty attached to the MDRD-eGFR results. Also, the large cohorts enhance the statistical significance that may not be of the same importance in clinical practice. Therefore, the algorithms seem reasonably transferable between populations at least in the reporting interval and excluding the lowest and highest age group.

Table 4. Medians of eGFR II and difference to the corresponding MDRD-eGFR values (Table 1). Medians and differences are expressed in $\mathrm{mL} /\left(\min \times 1.73 \mathrm{~m}^{2}\right)$. Non-significant differences in bold.

\begin{tabular}{|c|c|c|c|c|c|c|c|c|c|}
\hline Age-group & \multicolumn{2}{|c|}{ Males } & \multicolumn{2}{|c|}{ Females } & & \multicolumn{2}{|c|}{ Males } & \multicolumn{2}{|c|}{ Females } \\
\hline SE & Median & Difference & Median & Difference & UK & Median & Difference & Median & Difference \\
\hline $21-30$ & 95.9 & 9.2 & 108.4 & 4.1 & & 94.2 & 11.0 & 98.0 & 6.9 \\
\hline $31-40$ & 85.1 & 5.8 & 95.4 & -0.2 & & 83.5 & 6.4 & 86.8 & 3.0 \\
\hline $41-50$ & 76.8 & 2.8 & 86.8 & -3.1 & & 75.9 & 3.5 & 78.9 & -0.10 \\
\hline $51-60$ & 71.2 & 0.8 & 80.3 & -5.4 & & 69.3 & 1.6 & 73.0 & -1.9 \\
\hline $61-70$ & 66.0 & -0.4 & 74.6 & -5.8 & & 64.1 & 0.7 & 67.7 & -2.9 \\
\hline $71-80$ & 59.3 & -0.2 & 65.8 & -4.9 & & 58.7 & 0.2 & 61.9 & -2.8 \\
\hline $81+$ & 54.4 & -0.3 & 58.8 & -3.7 & & 53.7 & 0.2 & 56.5 & -2.6 \\
\hline $90+$ & 48.9 & 0.3 & 52.7 & -2.6 & & & & & \\
\hline
\end{tabular}

The course of changes of S-Creatinine, Pt-Iohexol and eGFR II over the studied ages is shown in figure 2. The changes in Pt-Iohexol and inverted S-Creatinine follow each other closely as does the eGFR II. The conversion of the S-Creatinine by any of the algorithms we tested does thus not contribute to a more effective understanding of the kidney function.
The uncertainty of the factors and exponents of the original MDRD-eGFR algorithm is not known to the authors, however, data from the present study (Table 3), provides an estimate of the combined uncertainty of $15 \%$ for the results of the eGFR II. This may be applicable to the original MDRD-eGFR and 
indicates an expanded uncertainty of about 20 $\mathrm{mL} /\left(\min \times 1.73 \mathrm{~m}^{2}\right)(\mathrm{k}=2)$ at S-Creatinine $100 \mu \mathrm{mol} / \mathrm{L}$, equating to an eGFR level of about $60 \mathrm{~mL} /(\min \times 1.73$ $\mathrm{m}^{2}$ ). It is interesting to note that the variation that is claimed acceptable by the K/DOQI [10] at MDRD-eGFR $60 \mathrm{~mL} /\left(\min \times 1.73 \mathrm{~m}^{2}\right)$, is $42-78 \mathrm{~mL} /(\min$ $\left.x 1.73 \mathrm{~m}^{2}\right)$ which is compatible with our uncertainty calculations $\left(40-80 \mathrm{~mL} /\left(\min \times 1.73 \mathrm{~m}^{2}\right)\right)$. Since the calculated uncertainty corresponds to an intralaboratory uncertainty it is an underestimate of the interlaboratory uncertainty that should be the basis for a recommendation. The uncertainty of S-Creatinine is about $14 \mu \mathrm{mol} / \mathrm{L}$ or one third.

Therefore the use of MDRD-eGFR in diagnosis may be misleading and the large uncertainty is a disadvantage in monitoring.

The ROC data (Figure 4) shows that S-Creatinine and MDRD-eGFR perform similarly. S-Creatinine results, however, are associated with a much smaller uncertainty than the MDRD-eGFR and accordingly will allow identifying smaller changes in the kidney function.

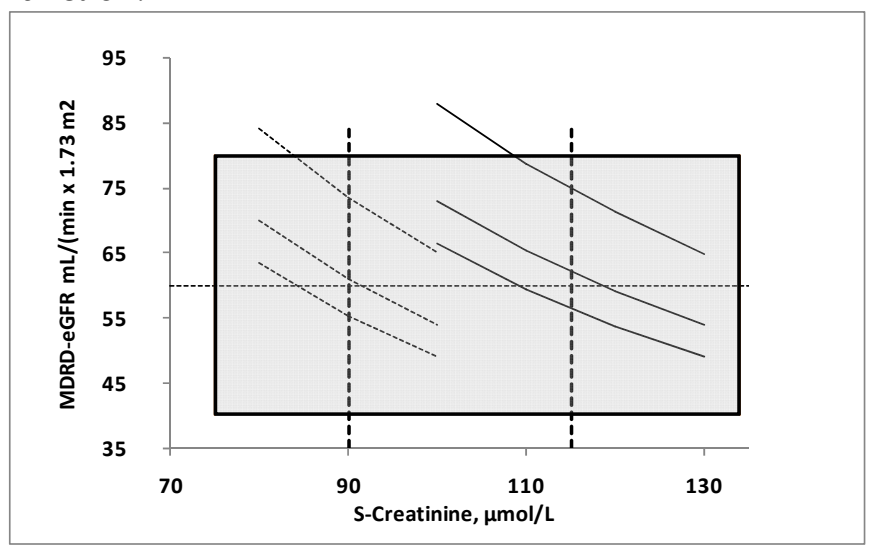

Figure 4. Relation between MDRD-eGFR (mL/(min x 1.73 $\left.\mathrm{m}^{2}\right)$ ) and S-Creatinine ( $\left.\mu \mathrm{mol} / \mathrm{L}\right)$. Curves represent (from upper) ages 20, 50 and 80 years. Females to the left. Vertical dashed lines are suggested creatinine cut-offs. The shaded area represents the uncertainty of the MDRD-eGFR based on the present study.

Many authors claim that S-Creatinine is a poor marker for glomerular filtration rate [20]. It is therefore an intriguing thought that a simple algorithm that essentially is based on a negative exponent $(-1,154)$ of S-Creatinine (equal to 0,87 at S-Creatinine $100 \mu \mathrm{mol} / \mathrm{L}$ ) and an age compensating factor of about $0,45(0,54$ at 20 years and 0,42 at 80 years) and a magnifying constant factor (174-186) will drastically change the diagnostic power of the measurand. On the contrary, the algorithm will increase the uncertainty of the result and thus the diagnosis. The uncertainty found in our derived algorithm transferred to a $95 \%$ level of confidence $\left( \pm 18 \mathrm{~mL} /\left(\min \times 1.73 \mathrm{~m}^{2}\right)\right)$ is almost equal to the analytical goal by K/DOQI $\pm 30 \%( \pm 18 \mathrm{~mL} /(\mathrm{min} x$ $\left.1.73 \mathrm{~m}^{2}\right)$ at $\left.60 \mathrm{~mL} /\left(\min \times 1.73 \mathrm{~m}^{2}\right)\right)$ [10]. The implication of this, as illustrated in figure 4 is that a S-Creatinine cut-off of $90 \mu \mathrm{mol} / \mathrm{L}$ and $110 \mu \mathrm{mol} / 1$ for females and males, respectively, would correspond to a eGFR of $60 \mathrm{~mL} /\left(\min \times 1.73 \mathrm{~m}^{2}\right)$. Measurement of S-Creatinine is also easier to standardize than algorithms based on regression analysis.

\section{Conclusion}

Transformation of S-Creatinine to eGFR according to the MDRD-eGFR algorithm or a similarly derived algorithm does not compensate for the physiological differences between age groups and gender. A common cut-off for additional examinations, investigations or diagnosis does thus not seem justified, i.e. we either have to fully compensate for the effects of gender and age or have different cut-offs for the different age groups and gender. The present study does not support an assumed advantage of factorizing S-Creatinine to create a number that superficially resembles that of iohexol clearance. Considering the low LR, the pretest probability (prevalence of disease) needs to amount to about $20 \%$ or higher for either quantity as a single test to be of diagnostic value.

\section{Acknowledgements}

This study was financed in full by the hospitals as routine parts of their quality improvement efforts.

\section{Conflict of interests}

The authors have declared that no conflict of interest exists.

\section{References}

1. Rainey PM. Automatic reporting of the estimated glomerular Filtration Rate - Jumping the gun? Clin Chem 2006;52:2184-87.

2. Levey AS, Stevens LA, Hostetter T. Automatic reporting of estimated glomerular filtration rate - Just what the doctor ordered. Clin Chem 2006;52:2188-2193.

3. National Kidney Foundation. K/DOQI clinical practice guidelines for chronic kidney disease: evaluation, classification, and stratification. Kidney Disease Outcome Quality Initiative. Am J Kidney Dis. 2002;39:S1-246.

4. Levey AS, Coresh J, Balk E, Kausz AT, Levin A, Steffes MW et al.. National Kidney Foundation Practice Guidelines for Chronic KidneyDisease: Evaluation, Classification, and Stratification. Ann Intern Med 2003;139:137-47..

5. Hostetter TH, Lising M. National Kidney Disease Education Program. Am J Kidney Disease 2002;39(Suppl 1): s17-s223.

6. Eriksson C-G, Kallner A. Glomerular filtration rate: a comparison between Cr-EDTA clearance and a single sample technique with a non-ionic contrast agent. Clin Biochem 1991;24:261-264.

7. Brandstrom E, Grzegorczyk A, Jacobsson L, Friberg P, Lindahl A,Aurell M. GFR measurement with iohexol and 51Cr-EDTA. A comparison of the two favoured GFR markers in Europe Nephrol Dial Transplant, 1998;13:1176-1182. 
8. BIPM, IEC, IFCC, ISO, IUPAC, IUPAP, OIML. Guide to the expression of uncertainty in measurement. Geneva: ISO. 1993.

9. Fraser CG. Biological variation: From principles to practice. Washington DC USA: AACC press. 2001.

10. Stevens LA, Coresh J, Feldman HI, el al. Evaluation of the modification of the diet in renal disease study equation in a large diverse population. J Am Soc Nephrol; 2007;18:2749-2757.

11. Kallner A, Ayling PA, Khatami Z. How does MDRD-eGFR perform compared to S-Creatinine in routine healthcare? Poster Clin Chem 2007;53(6):A119

12. Lippi G, Salvagno GL ,Guidi GC. Is estimated glomerular filtration rate suitable for stratification of chronic kidney disease? Scand J Clin Lab Invest. accepted for publication.

13. Payne RB. Creatinine clearance: a redundant clinical investigation. Ann Clin Biochem 1986;23:241-250.

14. Myers GL, Miller G, Coresh J, Fleming J, Greenberg N, Greene T, et al. for the National Kidney Disease Education Program Laboratory Working Group Recommendations for Improving Serum Creatinine Measurement: A Report from the Laboratory Working Group of the National Kidney Disease Education Program. Clin Chem 2006;52:5-18.

15. Vicerey S, Stevens PE, Dalton RN, van Lente F, Lamb EJ. Does the ID-MS traceable MDRD equation work and is it suitable for use with compensated Jaffe and enzymatic creatinine assays? Nephrol Dial Transplant 2006;21:2439-45.

16. Miller JN, Miller JC. Statistics and Chemometrics for Analytical Chemistry. Harlow, UK: Perason Education Ltd. 2005.

17. Rule AD, Larson TS, Bergstralh EJ, Slezak JM, Jacobsen SJ, Cosio FG. Using serum creatinine to estimate glomerular filtration rate: accuracy in good health and in chronic kidney disease. Ann Intern Med 2004;141:929-37.

18. Lin J, Knight EL, Hogan ML, Singh AK. A comparison of prediction equations for estimating glomerular filtration rate in adults without kidney disease. J Am Soc Nephrol 2003;14:2573-80.

19. Khatami Z, Handley G, Narayanan K, Weaver JU. Applicability of estimated glomerular filtration rate in stratifying chronic kidney disease. Scand J Clin Lab Invest 2007;67:297-305.

20. MacGregor MS, Boag DE and Innes A. Chronic kidney disease: evolving strategies for detection and management of impaired renal function. Q J Med 2006;99:365-375. 